\begin{tabular}{|c|c|c|}
\hline & Int.J.Curr.Microbiol.App.Sci (2016) 5(12): 933-947 & ucMAs \\
\hline & International Journal of Current Microbiology and Applied Sciences & 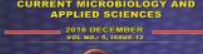 \\
\hline & ISSN: 2319-7706 Volume 5 Number 12 (2016) pp. 933-947 & \\
\hline $\begin{array}{l}\text { EXCELLENT } \\
\text { PUBLISHERS }\end{array}$ & & 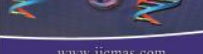 \\
\hline
\end{tabular}

Original Research Article

http://dx.doi.org/10.20546/ijcmas.2016.512.102

\title{
Probiotic, Prebiotic and Synbiotics in the Prevention of Lifestyle Disorders
}

\author{
Abhishek Chauhan ${ }^{1}$, Anuj Ranjan ${ }^{1}$, Rupesh Kumar Basniwal ${ }^{2}$ and Tanu Jindal ${ }^{1}$ \\ ${ }^{1}$ Amity Institute of Environmental Toxicology, Safety and Management, Amity University, \\ Sector-125, Noida, Uttar Pradesh, India \\ ${ }^{2}$ Amity Institute of Advanced Research and Studies, Amity University, Sector-125, \\ Noida, Uttar Pradesh, India \\ *Corresponding author
}

\begin{tabular}{|c|c|}
\hline & A B S T R A C T \\
\hline & \multirow{5}{*}{$\begin{array}{l}\text { Probiotics, prebiotics and synbiotics are the new concepts that have been developed to } \\
\text { amend the target gastrointestinal microflora balance required for growth and } \\
\text { development. Globally awareness about theprobiotic foods israpidly increasing and } \\
\text { consumers are now very much conscious about their health and the food that they eat. } \\
\text { Evidence for various beneficial roles of the intestinal microbiota in human health and } \\
\text { disease is expanding rapidly. Perturbation of the intestinal microbiota may lead to } \\
\text { chronic diseases such as autoimmune diseases, colon cancers, gastric ulcers, } \\
\text { cardiovascular disease, functional bowel diseases, and obesity. Several scientific } \\
\text { research studies rendering that the gut health in general is a key sector for maintaining } \\
\text { the overall health of people, whose microbiota plays a key role in metabolism and } \\
\text { nutrient absorption. A large of number of research organization are working on several } \\
\text { aspects including Genomics and bioinformatics of probiotic microorganisms to produce } \\
\text { multifunctional probiotic with one organisms or the mixture of several strains. Some } \\
\text { effects attributed to selected probiotics or prebiotics have been proved by clinical trials, } \\
\text { while others have been acquired on the basis of in vitro tests which need to be } \\
\text { replicated in vivo in order to be validated. In Indian context so far Yakult a big brand of } \\
\text { Probiotic foods sold in metro city against the huge increasing demands. Probiotic } \\
\text { microorganisms especially Lactobacillus and Bifidobacterium have been suggested to } \\
\text { be associated with a number of health benefits. The present review is a collection of } \\
\text { scientific information to enrich probiotic research and development internationally. }\end{array}$} \\
\hline Keywords & \\
\hline & \\
\hline & \\
\hline & \\
\hline
\end{tabular}

\section{Introduction}

In 1908, Dr Elie Metchnikoff, a Russian biologist first introduced the concept of probiotics. Probiotic terms derived from Greek words Pro (favor) and bios (life).Lilly and Stillwell in 1965 describe "substances secreted by one microorganism which stimulates the growth of another" and thus was contrasted with the term antibiotic. It may be because of this positive and general claim of definition that the term probiotic was subsequently applied to other subjects and gained a more general meaning. Probiotics are defined as live microorganisms which when administered in adequate amounts confer a health benefit on the host (FAO and WHO, 2002) Whereas Prebiotic are the food components which promote the growth and proliferation of useful bacteria in the digestive 
system. These Prebiotics are not easily digested by human and therefore indirectly very essential for life. Synbiotic probably a new term, it is a synergistic combination of Pre and Probiotic. The normal human digestive tract contains more than 400 different types of bacteria that promote a healthy digestive system. The global probiotic market generated US $\$ 15.9$ billion in 2008 and is expected to be worth US\$ 32.6 billion by 2014 with a compound annual growth rate of $12.6 \%$ from 2009 to 2014. On the other hand the probiotic product industry in India was estimated to be around Rs 20.6 million with a projected annual growth rate of $22.6 \%$ until 2015 (ICMR-DBT, 2011).The World Health Organization (WHO) estimates 8.1 million deaths occur yearly in children $(<5$ years of age) with diarrhea accounting for $14 \%$ of those deaths. Lactic acid bacteria such as lactobacilli are most commonly used as Probiotic bacteria bifidobacteria and other bacilli and certain yeast may also be used. Probiotic microorganisms (e.g. Lactobacillus rhamnosus GG, L. reuteri, bifidobacteria and certain strains of $L$. casei or the $L$. acidophilus-group) are used in Probiotic food, particularly fermented milk products, or have been investigated--as well as Escherichia coli strain Nissle 1917, certain enterococci (Enterococcus faecium SF68) (De Vrese and Schrezenmeir, 2008). Probiotic products are available in the form of Foods such as fermented milk, yoghurt and ice-cream. Powder, capsule and tablets as a dietary supplements and pharmaceutical formulations with specific therapeutic claims as a drug. Fermented milks are commonly included in many different national food dietary guidelines that include the dairy category (Antoine, 2014). Several studies in animal models and in human population have confirmed that the utilization of probiotics is effective in various medical conditions such as lactose intolerance, antibiotic-induced diarrhea, gastroenteritis, constipation, and genitourinary tract infections (Iannitti and Palmieri, 2010). Moreover, accumulating evidence suggests that the ingestion of probiotics may be able to play a preventive role in the onset of CRC (Rafter, 2004). This review is purely intended at providing an outline of the possible scientific information nationally as well as internationally. It is a cumulative effort to enrich students, researchers, Scientists and Technologists as far as basic to current applied information in this area.

\section{Probiotic microorganisms}

Probiotic cultures are described as live microbial feed supplements that improve intestinal microbial balance. The gastrointestinal tract is estimated to contain approximately 100 trillion microbial cells (Ramakrishna, 2014). The 'normal' gut microflora consists of bacterial species with morphological, physiological and genetic features that let it to colonize and multiply under particular conditions at certain sites, coexist with other colonizing microorganisms and competitively inhibit the growth of pathogenic bacteria. Microbial population of the gut is very sparse in the stomach due to the luminal acidity and vigorous peristalsis. The microbial population increases in density as we proceed from the duodenum $\left(10^{1}-10^{3}\right.$ organisms $/ \mathrm{ml})$ to jejunum $\left(10^{4}-10^{6}\right.$ organisms/ $\mathrm{ml})$ to ileum $\left(10^{5}-10^{7}\right.$ organisms $\left./ \mathrm{ml}\right)$. Microbial Concentration in the large intestine reaches $10^{11}-10^{12}$ organisms/g of stool (Ramakrishna, 2014). Some environmental factors such as diet and drugs can alter the composition of the resident microbiota, with consequent dysmicrobia and negative implications for the health of the individual. The colonic microflora is very rich and dominated by strict anaerobic bacteria such as Bacteroides spp., Fusobacterium spp., Clostridium spp, and many others (Uccello et $a l ., 2012)$. This is a particular concern, given 
thathigh levels (at least $10^{7}$ per gram or $\mathrm{ml}$ ) of live micro-organisms are recommended for probiotic products. Antoine in 2014 has reported that probiotics belongs to different phyla: the phylum firmicutes with the order Lactobacillacae, phylum actinobacteria and phylum proteobacteria. Scientists are in Search for new strain for the expansion of the products with novel strain on microorganisms.

\section{Intrinsic and Extrinsic Factor Affecting Probiotic Viability in Food}

Probiotic microorganisms are affected by several intrinsic and extrinsic factors. Therefore it is very much useful to study these factors in all stage of Probiotic food manufacturing. Following key factors have been described.

Temperature: Microorganisms are divided on the basis of temperature, hence it is very important factor that is directly affects the viability of Probiotic microorganisms. Therefore, the products (raw material, finished or any stage) are being stored at lower temperature instead of High. Several national and international guidelines are used to maintain the optimum temperature during the all stages of Probiotic manufacturing.

Oxygen: There are certain strains i.e. Bifidobacterium which are anaerobic and presence of oxygen can stop their growth and survival. The amount of oxygen sensitivity varies from one strain to another which can be studied practically. Obligate anaerobic Probiotic strains cannot survive but facultative strains i.e. Lactobacilli can tolerate more oxygen. Therefore, as per the national and international guidelines it is recommended that for Oxygen sensitive strains, it is desirable to diminish such process which involve aeration.
Water Content: It is also a very significant factor for probiotic survival in the foods. Water Moisture and osmotic stress are controlled through prerequisites. It is reported that when the moisture and water content of the product is higher, the survival of probiotic microorganisms would be lower. To achieve good quality and stability water content is kept as per the limitation of guidelines to avoid product drying.

pH: In food and dairy industry, $\mathrm{pH}$ of the product is controlled for long shelf life of probiotic products. There are certain important strains of microorganisms which produced organic acid as end product of carbohydrate fermentation and thereby accelerate the survival of probiotic microorganisms.

Freeze and Thawing: These factors directly affects of the survival of probiotic strains as freezing can be harmful and cells may become more vulnerable to environmental stress. Protectants are added in the culture before frozen or dried to prevent cell injury and damage. Other freezing methods can be adopted after validation studies.

Shear Force: Cell disruption and loss of viability may take place during production process such as high-speed blending or homogenization, Gram-positive bacteria due to thick cell wall are able to tolerate the shear forces.

Physiological State of Probiotic Microorganisms: It is also a very key factor as probiotic microbial stains have their own intrinsic tolerance to environmental condition. Moreover, conditions depends the maintenance of culture and their passage number at the time of preparation of porobiotic formulation and products. 
Synergistic Ingredient, Toxicity and Inhibition: Selection of carrier matrix should have synergistic interaction with probiotic microorganisms. Antimicrobial substance (Organic acid, salt, nitrates and preservative) can inhibit probiotic microorganisms during their storage. Certain antimicrobial effectiveness tests are recommended to determine the antimicrobial nature of the raw products. However, the ingredients must support to improve the growth of selected Probiotic strains.

Guidelines for the Evaluation of Probiotic in Food (adopted from Koshia and Sesikeran, 2014)

1. Strain Identification by phenotypic and genotypic methods (a) Genus, species, and Strain (b) Deposit strain in an internationally recognized collection.

2. Screening of Potential Probiotic strains (a) In Vitro Tests

3. In Vivo Studies in validated animal models for: (a) Safety (b) Efficacy

4. In Vivo study in humans for clinical evaluations (a) Phase 1 Safety (b) Phase 2 efficacy (c) Phase 3 Effectiveness.

5. Probiotics Foods: Labeling requirements (a) Genus, Species, Strain (b) Minimum viable numbers of Probiotics at the level at which efficacy is claimed and at the end of shelf life (c) Health Claims (d) Serving size for efficacy (d) Storage condition.

Probiotic Products and Producer (PPP): A number of microbial strains are now used for the preparation of probiotic dinks and other products. Name of the microbial strains, product brand and producer are given in the Table 1.
Product Brand with Mixture of Microbial Strains: Several manufacturer and R\&D organization are using the combination of microbial strains for new or better probiotic products. Some of the product brands are already developed (Table 2).

Benefits and Clinical Application: Probiotic microorganism's exhibits several immunological and non immunological benefits are given in the Table: 3 . It has been widely reported that the probiotic preparation and products are known to reduce or prevent several clinical disorders such as Cardiovascular disease, Colon cancer, Diarrhea, Prevention of acute diarrhea: Antibiotic-associated diarrhea: Radiationinduced diarrhea etc. Research studies throughout the world have been carried out to evaluate probiotic products against more human disorders. Many studies have shown that Probiotics can stimulate the immune system, decrease serum cholesterol, alleviate lactose intolerance, decrease diarrheal incidence, control infections. Some of the common disorders are describe as under: Probiotic bacteria release a variety of chemical compounds that are inhibitory to both gram-positive and gram negative bacteria. These include bacteriocins, sideropheres, lysozymes, proteases, hydrogenperoxides etc. Bacteriocins are proteinaceous compounds produced by a wide range of bacteria exhibiting antimicrobial activity against a select range of other bacteria (Jothi et al., 2012).

\section{Prebiotics}

Prebiotics are food ingredients which are indigestible in the upper GI tract and reach the colon to beneficially influence the host by selectively promoting the growth and/or activity of certain bacteria in the colon. Prebiotics are employed to promote both beneficial bacteria which are already established in the colon as well as externally 
administered probiotic bacteria. Prebiotics are also present in low amount in many fruits and vegetables including leeks, Jerusalem artichokes, chicory, onion, garlic, banana, and asparagus. All prebiotics are carbohydrates, and there are many different carbohydrates marketed world-wide as prebiotics, Oligosaccharides such as lactulose, galactooligo-saccharides, inulin, fructooligosaccharides, and other food carbohydrates are some of the well known examples of prebiotics (Soccol et al., 2010).

Product with recommended dose: It has been well said that the excess of everything is harmful, even high amount of salt become toxic therefore, dose of the product play a crucial role for better benefits. Several strains have been selected or under identification are being used in several products. The detail information is summarized in Table 4.

\section{Relevance of Probiotics in Aquaculture and Animal Feed}

Now a day, there has been a considerable interest in using some probiotic microorganisms and organic acids as an alternative to the use of antibiotics in feed. Animal feed companies and researchers have been looking for alternative products and strategies that can help to maintain animal gut health in order to preventor reduce the prevalence of pathogens in the food chain.

Table.1 Example of Probiotic strains in Products (adopted from World Gastroenterology Organisation Practice Guideline, 2008)

\begin{tabular}{|c|c|c|}
\hline Microbial Strains & Product Brand & Producer \\
\hline Bifidobacterium animalis DN 173010 & Activia & Danone/Dannon \\
\hline Bifidobacterium animalis subsp. lactis $B b-12$ & & Chr. Hansen \\
\hline Bifidobacterium breve & YakultBifiene & Yakult \\
\hline Bifidobacterium infantis 35624 & Align & Procter \& Gamble \\
\hline Bifidobacterium lactis HN019 (DR10) & Howaru Bifido & Danisco \\
\hline Bifidobacterium longum BB536 & & Morinaga Milk Industry \\
\hline Enterococcus LAB SF 68 & Bioflorin & Cerbios-Pharma \\
\hline Escherichia coliNissle 1917 & Mutaflor & Ardeypharm \\
\hline Lactobacillus acidophilus LA-5 & & Chr. Hansen \\
\hline Lactobacillus acidophilus NCFM & & Danisco \\
\hline Lactobacillus casei DN-114001 & Actimel, DanActive & Danone/Dannon \\
\hline Lactobacillus casei CRL431 & & Chr. Hansen \\
\hline Lactobacillus casei F19 & Cultura & Arla Foods \\
\hline Lactobacillus casei Shirota & Yakult & Yakult \\
\hline Lactobacillus johnsonii La1 (Lj1) & LC1 & Nestlé \\
\hline Lactococcus lactis L1A & Norrmejerier & \\
\hline Lactobacillus plantarum 299V & GoodBelly, ProViva & NextFoodsProbi \\
\hline Lactobacillus reuteri ATTC 55730 & Reuteri & BioGaia Biologics \\
\hline Lactobacillus rhamnosus ATCC 53013 (LGG) & Vifit and others & Valio \\
\hline Lactobacillus rhamnosus LB21 & Verum & Norrmejerier \\
\hline Saccharomyces cerevisiae (boulardii) lyo & $\begin{array}{l}\text { DiarSafe, Ultralevure, } \\
\text { and others }\end{array}$ & $\begin{array}{l}\text { Wren Laboratories, } \\
\text { Biocodex, and others }\end{array}$ \\
\hline
\end{tabular}


Table.2 Example of Probiotic strains in Products tested as a mixture (adopted from World Gastroenterology Organisation Practice Guideline, 2008)

\begin{tabular}{|l|l|l|}
\hline \multicolumn{1}{|c|}{$\begin{array}{c}\text { Microbial Strains } \\
\text { Tested as a Mixture }\end{array} \quad$ Product Brand } & \multicolumn{1}{c|}{ Producer } \\
\hline $\begin{array}{l}\text { Lactobacillus acidophilus CL1285 } \\
\text { \&Lactobacillus casei Lbc80r }\end{array} \quad$ Bio K+ & Bio K+ International \\
\hline $\begin{array}{l}\text { Lactobacillus rhamnosus GR-1 } \\
\text { \&Lactobacillus reuteri RC-14 }\end{array} \quad$ FemDophilus & Chr. Hansen \\
\hline $\begin{array}{l}\text { VSL\#3 (mixture of 1 strain of Streptococcus } \\
\text { thermophilus, four Lactobacillus spp., \& } \\
\text { three Bifidobacterium spp. strains } \\
\text { VSL\#3 }\end{array}$ & Sigma-Tau & Pharmaceuticals, Inc. \\
\hline $\begin{array}{l}\text { Lactobacillus acidophilus CUL60 } \\
\text { \&Bifidobacterium bifidum CUL 20 }\end{array}$ & - & - \\
\hline $\begin{array}{l}\text { Lactobacillus helveticus R0052 } \\
\text { \&Lactobacillus rhamnosus R0011 }\end{array}$ & A'Biotica and others & Institut Rosell \\
\hline \begin{tabular}{l} 
Bacillus clausii strains O/C, NR, SIN, and T \\
\hline
\end{tabular} & Enterogermina & Sanofi-Aventis \\
\hline
\end{tabular}

Table.3 Benefits and Clinical Application (adopted from World Gastroenterology Organisation Practice Guideline, 2008)

\begin{tabular}{|l|l|}
\hline $\begin{array}{l}\text { Immunologic } \\
\text { benefits }\end{array}$ & $\begin{array}{l}\text { Activate local macrophages to increase antigen presentation to B lymphocytes and } \\
\text { increase secretory immunoglobulin A (IgA) production both locally and systemically } \\
\text {;Modulate cytokine profiles;Induce hyporesponsiveness to food antigens }\end{array}$ \\
\hline $\begin{array}{l}\text { Non } \\
\text { immunologic } \\
\text { benefits }\end{array}$ & $\begin{array}{l}\text { Digest food and compete for nutrients with pathogens; Alter local pH to create an } \\
\text { unfavorable local environment for pathogens; Produce bacteriocins to inhibit } \\
\text { pathogens; Scavenge superoxide radicals; Stimulate epithelial mucin production; } \\
\text { Enhance intestinal barrier function; Compete for adhesion with pathogens; Modify } \\
\text { pathogen-derived toxins }\end{array}$ \\
\hline $\begin{array}{l}\text { Clinical } \\
\text { Application }\end{array}$ & $\begin{array}{l}\text { Cardiovascular disease, Colon cancer, Diarrhea, Prevention of acute } \\
\text { diarrhea:Antibiotic-associated diarrhea:Radiation-induced diarrhea, Eradication of }\end{array}$ \\
& $\begin{array}{l}\text { Helicobacter pylori, Allergy, Hepatic encephalopathy, Inflammatory bowel disease } \\
\text { (IBD)Irritable bowel syndrome (IBS)Lactose malabsorption, Necrotizing } \\
\text { enterocolitis, Nonalcoholic fatty liver disease, Prevention of systemic infections }\end{array}$ \\
\hline
\end{tabular}


Table.4 Probiotics and Prebiotics in gastroenterology (adopted from World Gastroenterology Organisation Practice Guideline, 2008)

\begin{tabular}{|c|c|c|c|}
\hline Disorder & Product & Recommended dose & References \\
\hline \multirow{4}{*}{$\begin{array}{l}\text { Treatment of acute } \\
\text { infectious diarrhea in } \\
\text { children }\end{array}$} & L. rhamnosus GG & $10^{10}-10^{11} \mathrm{cfu}$, twice daily & Allen et al., 2004 \\
\hline & L. reuteri ATTC 55730 & $10^{10}-10^{11}$ cfu, twice daily & Allen et al., 2004 \\
\hline & $\begin{array}{l}\text { L. acidophilus }+B \text {. infantis } \\
\text { (Infloran strains) }\end{array}$ & $\begin{array}{l}10^{9} \text { cfu each, three times } \\
\text { daily }\end{array}$ & Lee et $a l ., 2001$ \\
\hline & S. cerevisiae (boulardii) lyo & $200 \mathrm{mg}$, three times daily & Allen et al., 2004 \\
\hline $\begin{array}{l}\text { Treatment of acute } \\
\text { infectious diarrhea in } \\
\text { adults }\end{array}$ & $\begin{array}{l}\text { Enterococcus faecium LAB } \\
\text { SF68 }\end{array}$ & $10^{8} \mathrm{cfu}$, three times daily & Allen et al., 2004 \\
\hline \multirow{3}{*}{$\begin{array}{l}\text { Prevention of antibiotic- } \\
\text { associated diarrhea in } \\
\text { children }\end{array}$} & S. cerevisiae (Boulardii) lyo & $250 \mathrm{mg}$, twice daily & Sazawal et al., 2006 \\
\hline & L. rhamnosus GG & $\begin{array}{l}10^{10} \text { cfu once or twice } \\
\text { daily }\end{array}$ & Sazawal et al., 2006 \\
\hline & $\begin{array}{l}\text { B. lactis Bb12+ } \\
\text { S. thermophiles }\end{array}$ & $\begin{array}{l}10^{7}+10^{6} \mathrm{cfu} / \mathrm{g} \text { of } \\
\text { formula }\end{array}$ & Sazawal et al., 2006 \\
\hline \multirow{6}{*}{$\begin{array}{l}\text { Prevention of antibiotic- } \\
\text { associated diarrhea in } \\
\text { adults }\end{array}$} & $\begin{array}{l}\text { Enterococcus faecium } L A B \\
\text { SF68 }\end{array}$ & $10^{8} \mathrm{cfu}$, twice daily & Lee et $a l ., 2001$ \\
\hline & S. cerevisiae (boulardii) lyo & $\begin{array}{l}1 \mathrm{~g} \text { or } 3 \times 10^{10} \text { cfu per } \\
\text { day }\end{array}$ & Sazawal et al., 2006 \\
\hline & L. rhamnosus $G G$ & $10^{10}-10^{11} \mathrm{cfu}$, twice daily & Sazawal et al., 2006 \\
\hline & $\begin{array}{l}\text { L. casei DN-114 001 in } \\
\text { fermented milk with L. } \\
\text { bulgaricus }+S . \\
\text { thermophiles }\end{array}$ & $10^{10} \mathrm{cfu}$, twice daily & Hickson et al., 2007 \\
\hline & $\begin{array}{l}\text { B. clausii (Enterogermina } \\
\text { strains) }\end{array}$ & $\begin{array}{l}2 \times 10^{9} \text { spores, three } \\
\text { times daily }\end{array}$ & Nista et al., 2004 \\
\hline & $\begin{array}{l}\text { L. acidophilus CL1285+ } \\
\text { L. casei Lbc80r }\end{array}$ & $5 \times 10^{10} \mathrm{cfu}$, once daily & $\begin{array}{l}\text { Beausoleil et } a l . \text {, } \\
2007\end{array}$ \\
\hline \multirow{4}{*}{$\begin{array}{ll}\text { Prevention } & \text { of } \\
\text { nosocomial diarrhea in } \\
\text { children }\end{array}$} & L. rhamnosus $G G$ & $10^{10}-10^{11} \mathrm{cfu}$, twice daily & Sazawal et al., 2006 \\
\hline & $\begin{array}{l}\text { B. lactis BB12+ } \\
\text { S. thermophiles }\end{array}$ & $\begin{array}{llll}10^{8}+10^{7} & \mathrm{cfu} / \mathrm{g} & \text { of } \\
\text { formula } & & & \\
\end{array}$ & Sazawal et al., 2006 \\
\hline & B. lactis BB12 & $10^{9} \mathrm{cfu}$, twice daily & Sazawal et al., 2006 \\
\hline & L. reuteri ATTC 55730 & $10^{9} \mathrm{cfu}$, twice daily & Sazawal et al., 2006 \\
\hline \multirow[t]{4}{*}{$\begin{array}{lll}\text { Prevention of } & \text { C. } \\
\text { difficile diarrhea } & \text { in } \\
\text { adults } & & \end{array}$} & $\begin{array}{l}\text { L. casei DN-114001 in } \\
\text { fermented milk with } \\
\text { L. bulgaricus }+ \\
\text { S. thermophiles }\end{array}$ & $10^{10} \mathrm{cfu}$, twice daily & Hickson et al., 2007 \\
\hline & $\begin{array}{l}\text { L. acidophilus }+ \text { B. bifidum } \\
\text { (Cultech strains) }\end{array}$ & $\begin{array}{l}2 \times 10^{10} \mathrm{cfu} \text { each, once } \\
\text { daily }\end{array}$ & $\begin{array}{lll}\text { Beausoleil et } a l ., \\
2007\end{array}$ \\
\hline & S. cerevisiae (boulardii) lyo & $2 \times 10^{10}$ cfu per day & Sazawal et al., 2006 \\
\hline & Oligofructose & $4 \mathrm{~g}$, three times per day & Lewis et al., 2005 \\
\hline
\end{tabular}




\begin{tabular}{|c|c|c|c|}
\hline \multirow{5}{*}{$\begin{array}{l}\text { Adjuvant therapy for } \mathrm{H} \text {. } \\
\text { pylori eradication }\end{array}$} & L. rhamnosus $G G$ & $6 \times 10^{9} \mathrm{cfu}$, twice daily & Tong et al., 2007 \\
\hline & $\begin{array}{l}\text { B. clausii (Enterogermina } \\
\text { strains) }\end{array}$ & $\begin{array}{l}2 \times 10^{9} \text { spores, three } \\
\text { times daily }\end{array}$ & Tong et al., 2007 \\
\hline & $\begin{array}{l}\text { AB yogurt with unspecified } \\
\text { lactobacilli and } \\
\text { bifidobacteria }\end{array}$ & $\begin{array}{l}5 \times 10^{9} \text { viable bac, twice } \\
\text { daily }\end{array}$ & Tong et al., 2007 \\
\hline & S. cerevisiae (boulardii) lyo & $1 \mathrm{~g}$ or $5 \times 10^{9} \mathrm{cfu}$ per day & Tong et al., 2007 \\
\hline & $\begin{array}{l}\text { L. casei DN-114 } 001 \text { in } \\
\text { fermented milk with L. } \\
\text { bulgaricus }+S . \\
\text { thermophiles }\end{array}$ & $10^{10} \mathrm{cfu}$, twice daily & Sýkora et al., 2005 \\
\hline $\begin{array}{l}\text { Reduces symptoms } \\
\text { associated with lactose } \\
\text { maldigestion }\end{array}$ & $\begin{array}{l}\text { Regular yogurt with } L . \\
\text { bulgaricus }+S . \\
\text { thermophilus }\end{array}$ & $\begin{array}{l}\text { Yogurt not heat-treated } \\
\text { after pasteurization } \\
\text { contains suitable cultures } \\
\text { to improve digestion of } \\
\text { the lactose in the yogurt }\end{array}$ & Montalto et al., 2006 \\
\hline \multirow{5}{*}{$\begin{array}{l}\text { Alleviates some } \\
\text { symptoms of irritable } \\
\text { bowel syndrome }\end{array}$} & B. infantis 35624 & $10^{8} \mathrm{cfu}$, once daily & $\begin{array}{l}\text { O’Mahony et al., } \\
2005\end{array}$ \\
\hline & L. rhamnosus $G G$ & $6 \times 10^{9} \mathrm{cfu}$, twice daily & $\begin{array}{l}\text { Gawronska et al., } \\
2007\end{array}$ \\
\hline & VSL\# 3 mixture & $\begin{array}{l}4.5 \times 10^{11} \text { cfu, twice } \\
\text { daily }\end{array}$ & Kim et al., 2005 \\
\hline & $\begin{array}{l}\text { L. rhamnosus } G G, L . \\
\text { rhamnosus } L C 705, B \text {. breve } \\
\text { Bb99, and } \\
\text { Propionibacterium } \\
\text { freudenreichii ssp. } \\
\text { Shermanii }\end{array}$ & $10^{10} \mathrm{cfu}$, once daily & Kajander et al., 2005 \\
\hline & $\begin{array}{l}\text { B. animalis DN-173 } 010 \text { in } \\
\text { fermented milk with L. } \\
\text { bulgaricus }+S . \\
\text { thermophiles }\end{array}$ & $10^{10} \mathrm{cfu}$, twice daily & Guyonnet et al., 2007 \\
\hline $\begin{array}{l}\text { Maintenance r of } \\
\text { remission of ulcerative } \\
\text { colitis }\end{array}$ & E. coli Nissle 1917 & $\begin{array}{l}5 \times 10^{10} \text { viable bac, twice } \\
\text { daily }\end{array}$ & Kruis et al., 2004 \\
\hline $\begin{array}{l}\text { Prevention and } \\
\text { maintenance of } \\
\text { remission in pouchitis }\end{array}$ & $\begin{array}{l}\text { VSL\# } 3 \text { mixture of } 8 \text { strains } \\
\text { (1 S. thermophilus, } 4 \\
\text { Lactobacillus, } 3 \\
\text { Bifidobacterium) }\end{array}$ & $\begin{array}{l}4.5 \times 10^{11} \mathrm{cfu}, \text { twice } \\
\text { daily }\end{array}$ & $\begin{array}{l}\text { Gionchetti et al., } \\
2003\end{array}$ \\
\hline \multirow{2}{*}{$\begin{array}{ll}\text { Treatment } & \text { of } \\
\text { constipation } & \end{array}$} & Lactulose & $20-40 \mathrm{~g}$ per day & Schumann, 2002 \\
\hline & Oligofructose & $>20$ g per day & Nyman, 2002 \\
\hline \multirow{2}{*}{$\begin{array}{l}\text { Prevention of } \\
\text { necrotizing enterocolitis } \\
\text { in preterm infants }\end{array}$} & $\begin{array}{l}\text { B. infantis, S. thermophilus, } \\
\text { and B. bifidum }\end{array}$ & $\begin{array}{l}0.35 \times 10^{9} \text { cfu each } \\
\text { strain, once daily }\end{array}$ & $\begin{array}{l}\text { Deshpandeet } \\
2007\end{array}$ \\
\hline & $\begin{array}{l}\text { L. acidophilus }+ \text { B. infantis } \\
\text { (Infloran strains) }\end{array}$ & $10^{9}$ cfu each, twice daily & $\begin{array}{l}\text { Deshpande } e t \\
2007\end{array}$ \\
\hline
\end{tabular}




\begin{tabular}{|c|l|c|c|}
\hline $\begin{array}{c}\text { Prevention of } \\
\text { postoperative infections }\end{array}$ & $\begin{array}{l}\text { Synbiotic 2000: 4 bacteria } \\
\text { strains and fibers including } \\
\text { the prebiotic inulin }\end{array}$ & $\begin{array}{c}10^{10} \mathrm{cfu}+10 \mathrm{~g} \text { fibers, } \\
\text { twice daily }\end{array}$ & Rayes et al., 2005 \\
\hline $\begin{array}{c}\text { Treatment of hepatic } \\
\text { encephalopathy }\end{array}$ & Lactulose & $45-90 \mathrm{~g}$ per day & Schumann, 2002 \\
\hline
\end{tabular}

Table.5 Relevance of Probiotics in Aquaculture and Animal Feed(adopted from Soccol1 et al. 2010)

\begin{tabular}{|c|c|c|c|}
\hline Probiotic strain & Application & Probiotic effects & References \\
\hline $\begin{array}{l}\text { Bacillus subtilis, } \\
\text { Bacillus licheniformis }\end{array}$ & $\begin{array}{l}\text { shrimp } \\
\text { production }\end{array}$ & $\begin{array}{l}\text { Reduce stress, improve health, } \\
\text { the quality of water, clean } \\
\text { effluent water, } \\
\text { control pathogenic bacteria and } \\
\text { their virulence, stimulate the } \\
\text { immune } \\
\text { system, improve gut flora, } \\
\text { substitute antibiotics, improve } \\
\text { growth }\end{array}$ & $\begin{array}{l}\text { Decamp and } \\
\text { Moriarty 2006; } \\
\text { Moriarty et al. } \\
2005\end{array}$ \\
\hline $\begin{array}{l}\text { Bacillus spp. and } \\
\text { yeasts }\end{array}$ & $\begin{array}{l}\text { mollusc } \\
\text { production }\end{array}$ & $\begin{array}{l}\text { minimize diseases caused } \\
\text { by Vibrio spp. and Aeromonas } \\
\text { spp., which results in mollusc } \\
\text { mortality }\end{array}$ & $\begin{array}{l}\text { Watson et al. } \\
2008\end{array}$ \\
\hline Clostridium spp. & $\begin{array}{l}\text { freshwater } \\
\text { fish feed }\end{array}$ & $\begin{array}{l}\text { Produces digestive enzymes, } \\
\text { which facilitate feed utilization } \\
\text { and } \\
\text { digestion, antibacterial activity } \\
\text { against pathogenic } \\
\text { microorganisms }\end{array}$ & $\begin{array}{l}\text { Bairagi et al. } \\
2002\end{array}$ \\
\hline $\begin{array}{l}\text { Bacillus spp., } \\
\text { Saccharomyces } \\
\text { cerevisiae }\end{array}$ & aquaculture & $\begin{array}{l}\text { improve water quality and } \\
\text { interaction with phytoplankton, } \\
\text { possess } \\
\text { adhesion abilities, produce } \\
\text { bacteriocins, provide } \\
\text { immunostimulation }\end{array}$ & $\begin{array}{l}\text { Verschuere } e t \\
\text { al.2000 }\end{array}$ \\
\hline $\begin{array}{l}\text { Bacillus spp., } \\
\text { S. cerevisiae }\end{array}$ & Aquaculture & $\begin{array}{l}\text { stimulate the growth of } \\
\text { microalgae that produce organic } \\
\text { extracts capable } \\
\text { of inhibiting pathogens and } \\
\text { vibrios, then some microalgae } \\
\text { species produce } \\
\text { the antibiotic thiotropocin } \\
\text { against some pathogens }\end{array}$ & $\begin{array}{l}\text { Naviner } \text { et al. } \\
1999 ; \\
\text { Kawano et al } \\
1997\end{array}$ \\
\hline S. cerevisiae & aquaculture & immunostimulatory activity, & Verschuere $e t$ \\
\hline
\end{tabular}




\begin{tabular}{|c|c|c|c|}
\hline & & $\begin{array}{l}\text { produces inhibitory substances } \\
\text { against } \\
\text { pathogens }\end{array}$ & $a l .2000$ \\
\hline $\begin{array}{l}\text { Bifidobacterium } \\
\text { longum, } \\
\text { L. plantarum }\end{array}$ & chicken feed & $\begin{array}{l}\text { produce antimicrobial } \\
\text { substances against pathogens } \\
\text { such as Campylobacter }\end{array}$ & $\begin{array}{l}\text { Santini et al. } \\
2010\end{array}$ \\
\hline $\begin{array}{l}\text { Pediococcusacidilactici, } \\
\text { Lactococcuslactis, } \\
\text { L. casei, } \\
\text { Enterococcus faecium }\end{array}$ & weaned piglet & $\begin{array}{l}\text { stimulate animal growth, reduce } \\
\text { coliform counts by the } \\
\text { production of } \\
\text { antimicrobial metabolites }\end{array}$ & $\begin{array}{l}\text { Guerra et al. } \\
2007\end{array}$ \\
\hline S. cerevisiae & $\begin{array}{l}\text { lactating } \\
\text { ruminants }\end{array}$ & $\begin{array}{l}\text { facilitates increased } \\
\text { mobilization of body reserves, } \\
\text { increases milk fatty } \\
\text { acid production }\end{array}$ & $\begin{array}{l}\text { Reverdin et al. } \\
1996\end{array}$ \\
\hline S. cerevisiae & camel feed & $\begin{array}{l}\text { increases total mass gain and } \\
\text { improves feed utilization }\end{array}$ & $\begin{array}{l}\text { Mohamed et al. } \\
2009\end{array}$ \\
\hline S. cerevisiae & buffalo feed & increases digestion of cellulose & $\begin{array}{l}\text { Kumar et al. } \\
1994\end{array}$ \\
\hline Pediococcusacidilactici & broiler chickens & $\begin{array}{l}\text { improves performance, reduces } \\
\text { serum cholesterol }\end{array}$ & $\begin{array}{l}\text { Alkhalf et al. } \\
2010\end{array}$ \\
\hline $\begin{array}{l}\text { Lactobacillus, } \\
\text { Bifidobacterium, } \\
\text { Streptococcus, } \\
\text { Enterococcus ssp. }\end{array}$ & layer hens & reduces mortality & $\begin{array}{l}\text { Yörük et al. } \\
2004\end{array}$ \\
\hline L. sporogenes & broiler chickens & $\begin{array}{l}\text { reduces serum total cholesterol } \\
\text { and triglycerides }\end{array}$ & Panda et al. 2006 \\
\hline Lactobacillus ssp. & chicken feed & immunomodulating properties & $\begin{array}{l}\text { Koenen et al. } \\
2004\end{array}$ \\
\hline $\begin{array}{l}\text { Lactobacillus spp., } \\
\text { Bacillus spp. }\end{array}$ & poultry feed & $\begin{array}{l}\text { reduces zoonosis in poultry } \\
\text { meat }\end{array}$ & $\begin{array}{l}\text { Santini et al. } \\
2010\end{array}$ \\
\hline L. reuteri LPB P01-001 & swine feed & $\begin{array}{l}\text { mass gain, antimicrobial } \\
\text { activity against E. coli and S. } \\
\text { aureus }\end{array}$ & $\begin{array}{l}\text { Pancheniak, and } \\
\text { Soccol, 2005 }\end{array}$ \\
\hline $\begin{array}{l}\text { Enterococcus faecalis, } \\
\text { E. faecium }\end{array}$ & canine feed & $\begin{array}{l}\text { bacteriocin-like inhibitory } \\
\text { substances, antimicrobial } \\
\text { activity against } \\
\text { Gram(+) bacteria, colonize } \\
\text { transiently }\end{array}$ & $\begin{array}{l}\text { Strompfová et al. } \\
2004\end{array}$ \\
\hline
\end{tabular}

The use of probiotics and commercial products containing probiotics in aquaculture (e.g. shrimp production). In 2000 Verschuere and his co workers suggested a new definition 
of a probiotic for aquatic environments: 'a live microbial adjunct which has a beneficial effect on the host by modifying the hostassociated or ambient microbial community, by ensuring improved use of the feed or enhancing its nutritional value, by enhancing the host's response towards disease, or by improving the quality of its ambient environment', or that 'a probiotic is an entire microorganism or its components that are beneficial to the health of the host' (Irianto and Austin, 2002).

Quality Control and Quality Assurance: It is very utmost requirement that all the probiotic products must manufactured under Good manufacturing practices (GMP). It has also been reported that there are several microorganisms which can spoil the probiotic formulation and products, therefore at each level control of hazards and their detection must be carried out as per the available guidelines for probiotics. Assurance of raw material and other key parameters is equally significant for maintaining the quality of products in order to achieve increased demands of probiotics.

Conclusion and Path Forward: It can be concluded that prebiotics have great potential as agents to improve or maintain a balanced intestinal micro flora to enhance health and wellbeing. They can be incorporated into many foodstuffs. Thorough comparative studies will allow intelligent choices in incorporating prebiotics into functional foods and that should increase confidence among consumers and regulatory authorities. Prebiotics will be a unique tool to create, both in experimental animals and in humans, colonic micro flora with controlled compositions that will then be correlated with specific physiological conditions. Variety of probiotics studies are now on fast track across the age spectrum, in an ever increasing range of diseases, using a variety of routes of administration. The future is going to be challenging but promising, since tools for probiotic research are now available. Much work has already been accomplished to help us understand probiotics and the manner in which they function. Therefore the field of probiotics, prebiotics and synbiotics may potentially open a new branch of science.

\section{Acknowledgement}

Author is very thankful to Yakuly India Microbiota and Probiotic Science Foundation, India for scientific information. Author also very much thankful to Dr. Ashok K. Chauhan, Founder President, Amity University for his continuous support and motivation.

\section{References}

Alkhalf, A., Alhaj, M., Homidan, I.A. 2010. Influence of probiotic supplementation on blood parameters and growth performance in broiler chickens, Saudi J. Biol Sci., 17, 219-225.

Allen, S.J., Okoko, B., Martinez, E., Gregorio, G., Dans, L.F. 2004. Probiotics for treating infectious diarrhoea. Cochrane Database Syst Rev, (2):CD003048.

Antoine, J.M. 2014. Cross-talks between Dietary Probiotics and Gut Microbiota. Probiotics in the prevention of Lifestyle Disorders, Elsevier, ISBN 978-81-3123611-6.28-41.

Bairagi, A., Ghosh, K.S., Sen, S.K., Ray, A.K. 2002. Enzyme producing bacterial flora isolated from fish digestive tracts, Aquacult. Int., 10, 109-121.

Beausoleil, M., Fortier, N., Guénette, S., et al. 2007. Effect of a fermented milk combining Lactobacillus acidophilus C11285 and Lactobacillus casei in the prevention of antibiotic-associated diarrhea: a randomized, double-blind, 
placebo-controlled trial. Can. J. Gastroenterol., 21: 732-6.

Chattterjee, R., Sinha, S., Aggarwal, S., Dimri, A.G., Singh, D., Goyal, P., Chauhan, A., Aggarwal, M.L. and Chacko, K.M. 2012. Studies on susceptibility and resistance patterns of various E. coli isolated from different water samples against clinically significant antibiotics. Int. J. Bioassays, 01(11): 156-161.

Chauhan, A. and Goyal, P. 2013. Isolation and Identification of Escherichia coli from various foodstuffs and their resistance against clinically significant antibiotics, J. Adv. Biol., 2: 45-53.

Chauhan, A., Goyal, P., Aggarwal, M.L., Chacko, K.M. 2013. Prevalence and antibiotic resistance of Bacillus strains isolated from various food stuffs. $J$. Biomed. Pharma. Res., 2(3): 08-16.

Chauhan, A., Goyal, P., Varma, A., Jindal, T. 2015. Microbiological evaluation of drinking water sold by roadside vendors of Delhi, India. Appl. Water Sci., DOI 10.1007/s13201-015-0315.

Chauhan, A., Goyal, P., Verma, A. and Jindal, T. 2015. In -Vitro Antibiotic Resistance and Heavy Metal Tolerance Patterns of Gram-Positive and Gram-Negative Bacteria Isolated From Effluent Treated Water of Delhi, India. J. Curr. Pharma Res., 5(2): 1449-1458.

Chauhan, A., Pandey, V., Chacko, K.M. \& Khandal, R.K. 2010. Antibacterial activity of raw and processed honey. Electronic J. Biol., 5(3): 58-66.

De Vrese, M., Schrezenmeir, J. 2008. Probiotics, Prebiotics, and Synbiotics. Adv Biochem Eng Biotechnol., 111: 166.

Decamp, O., Moriarty, D.J.W. 2006. Probiotics as alternative to antimicrobials: Limitations and potential, World Aquaculture, 37, 6062.
Deshpande, G., Rao, S., Patole, S. 2007. Probiotics for prevention of necrotising enterocolitis in preterm neonates with very low birthweight: a systematic review of randomised controlled trials. Lancet, 369: 1614-20.

Garima, Chauhan, A., Goyal, P., and Kaushik, P. 2013. Antibacterial and anticandidal screening of extracellular and intracellular extracts of Phormedium, a cyanobacterium. Int. J. Chem. And Life Sci., Vol. 2(3), 1107-1111.

Gawronska, A., Dziechciarz, P., Horvath, A., Szajewska, H. 2007. A randomized double-blind placebo-controlled trial of Lactobacillus GG for abdominal pain disorders in children. Aliment Pharmacol. Ther., 25: 177-84.

Gionchetti, P., Rizzello, F., Helwig, U., et al. 2003. Prophylaxis of pouchitis onset with probiotic therapy: a double-blind, placebo-controlled trial. Gastroenterol., 124: 1202-9.

Goyal, P., Khanna, A., Chauhan, A., Garima, and Kaushik, P. 2008. In Vitro Evaluation of Crude Extracts of Catharanthusroseus for Potential Antibacterial Activity. Int. J. Green Pharm., Vol.2, Issue 3, 176-181.

Guerra, N.P., P. Bernárdez, P.F., Méndez, J., Cachaldora, P., Castro, L.P. 2007. Production of four potentially probiotic lactic acid bacteria and their evaluation as feed additives for weaned piglets, Anim. Feed Sci. Technol., 134: 89-107.

Guidelines for the evaluation of Probiotics in Food. Report of Joint FAO/WHO working group on drafting Guidelines for the evaluation of Probiotics. London Ontario, Canada. 2002.

Gupta, C.P., Batra, R., Chauhan, A., Goyal, P. and Kaushik, P. 2009. Antibacterial activity and TLC bio autography of Ocimumbasilicum L. against pathogenic bacteria. J. Pharm. Res., Vol.2, Issue 3, 407-409. 
Guyonnet, D., Chassany, O., Ducrotte, P., et al. 2007. Effect of a fermented milk containing Bifidobacterium animalis DN-173 010 on the health-related quality of life and symptoms in irritable bowel syndrome in adults in primary care: a multicentre, randomized, doubleblind, controlled trial. Aliment Pharmacol. Ther., 26: 475-86.

Hickson, M., D'Souza, A.L., Muthu, N., et al. 2007. Use of probiotic Lactobacillus preparation to prevent diarrhoea associated with antibiotics: randomised double blind placebo controlled trial. BMJ, 335(7610): 80.

Iannitti, T., Palmieri, B. 2010. Therapeutical use of probiotic formulations in clinical practice. Clin. Nutr., 29: 701-725.

ICMR-DBT. 2011. Guidelines for Evaluation of Probiotics In Food, Director General Indian Council of Medical Research New Delhi - 110029.

Irianto, A., Austin, B. 2002. Probiotics in aquaculture, J. Fish Dis., 25, 633-642.

Jothi, V.V., Anandapandian, K.T.K. and Shankar, T. 2012. Bacteriocin production by probiotic bacteria from curd and its field application to poultry Arch. Appl. Sci. Res., 4(1): 336-347.

Kajander K, Hatakka K, Poussa T, Farkkila M, Korpela R 2005 A probiotic mixture alleviates symptoms in irritable bowel syndrome patients: a controlled 6-month intervention. Aliment PharmacolTher 22:387-94.

Kaushik, P., Garima, Chauhan, A., and Goyal, P. 2009. Screening

Lyngbyamajuscula for potential antimicrobial activity and HPTLC analysis of active methanolic extract. $J$. Pure and Appl. Microbiol., Vol.3, No. 1, 169-174.

Kaushik, P., Goyal, P., Chauhan, A., and Chauhan, G. 2010. In Vitro Evaluation of Antibacterial Potential of Dry Fruit Extracts of Elettariacardamomum
Maton ChhotiElaichi). Iranian J. Pharmaceutical Res., 9(3), 287-292.

Kawano, Y., Nagawa, Y., Nakanishi, H., Nakajima, H., Matsuo, M., Higashihara, T. 1997. Production of thiotropocin by a marine bacterium, Caulobacter sp. and its antimicroalgal activites, J. Marine Biotechnol., 5: 225-229.

Kim, H.J., Vazquez Roque, M.I., Camilleri, M., et al. 2005. A randomized controlled trial of a probiotic combination VSL\# 3 and placebo in irritable bowel syndrome with bloating. Neurogastro enterol. Motil., 17:687-96.

Koenen, M.E., Hulst, R.V.D., Leering, M., Jeurissen, S.H., Boersma, W.J. 2004 Development and validation of a new in vitro assay for selection of probiotic bacteria that express immunestimulating properties in chickens in vivo, FEMS Immunol. Med. Microbiol., 40, 119-127.

Koshia, H.G., Sesikeran, B. 2014. The Regulatory Scence: Standardizing Probiotics in India. Probiotics in the prevention of Lifestyle Disorders, Elsevier, ISBN 978-81-312-3611-6.100112.

Kruis, W., Fric, P., Pokrotnieks, J., et al. 2004. Maintaining remission of ulcerative colitis with the probiotic Escherichia coli Nissle 1917 is as effective as with standard mesalazine. Gut, 53: 1617-23.

Kumar, U., Sareen, V.K., Singh, S. 1994. Effect of Saccharomyces cerevisiae yeast culture supplement on ruminal metabolism in buffalo calves given a high concentrate diet, Anim. Prod., 59, 209.

Lee, M.C,. Lin, L.H., Hung, K.L., Wu, H.Y. 2001. Oral bacterial therapy promotes recovery from acute diarrhea in children. Acta Paediatr Taiwan, 42: 301-5. 
Lewis, S., Burmeister, S., Brazier, J. 2005. Effect of the prebiotic oligofructose on relapse of Clostridium difficileassociated diarrhea: a randomized, controlled study. Clin. Gastroenterol. Hepatol., 3: 442-8.

Lilly, D.M., Stillwell, R.H. 1965. Probiotics. Growth promoting factors produced by micro-organisms. Sci., 147: 747-8.

Mohamed, M.I., Maareck, Y.A., Magid Soha, S.A., Awadalla, I.M. 2009. Feed intake, digestibility, rumen fermentation and growth performance of camels fed diets supplemented with a yeast culture or zinc bacitracin, Anim. Feed Sci. Technol., 149, 341-345.

Montalto, M., Curigliano, V., Santoro, L., et al. 2006. Management and treatment of lactose malabsorption. World $J$. Gastroenterol., 12:187-91.

Moriarty, D.J.W., Decamp, O., Lavens, P. 2005. Probiotics in aquaculture, AQUA Culture Asia Pacific Magazine, 14-16.

Naviner, M., Bergé, J.P., Durand, P., Le Bris, H. 1999. Antibacterial activity of the marine diatom Skeletonemacostatum against aquacultural pathogens, Aquaculture, 174, 15-24.

Nista, E.C., Candelli, M., Cremonini, F., et al. 2004. Bacillus clausii therapy to reduce side-effects of anti-Helicobacter pylori treatment: randomized, double-blind, placebo controlled trial. Aliment Pharmacol Ther., 20: 1181-8.

Nyman, M. 2002. Fermentation and bulking capacity of indigestible carbohydrates: the case of inulin and oligofructose. $\mathrm{Br}$. J. Nutr., 87(Suppl 2):S163-8.

O’Mahony, L., McCarthy, J., Kelly, P., et al. 2005. Lactobacillus and Bifidobacterium in irritable bowel syndrome: symptom responses and relationship to cytokine profiles. Gastroenterol., 128: 541-51.

Pancheniak, E., Soccol, C.R. 2005. Isolation, selection, biochemical characterization for production and evaluation of probiotic potential of L. reuteri LPB P01-001 in swines, PhD Thesis, Food Technology Program, Federal University of Parana, Curitiba, Brazil in Portuguese).

Panda, A.K,. Rao, S.V.R., Rafu, M.V.L., Sharma, S.R. 2006. Dietary supplementation of Lactobacillus sporogenes on performance and serum biochemico-lipid profile of broiler chickens, J. Poultry Sci., 43, 235-240.

Plummer, S., Weaver, M.A., Harris, J.C., et al. 2004. Clostridium difficile pilot study: effects of probiotic supplementation on the incidence of Clostridium difficile diarrhoea. Int. Microbiol., 7: 59-62.

Rafter, J. 2004. The effects of probiotics on colon cancer development. Nutr. Res. Rev., 17: 277-284

Ramakrishna, B.S. 2014. Gut Microbiota in Indians and the effect of probiotics. Probiotics in the prevention of Lifestyle Disorders, Elsevier, ISBN 978-81-3123611-6.14-27.

Rayes, N., Seehofer, D., Theruvath, T., et al. 2005. Supply of pre- and probiotics reduces bacterial infection rates after liver transplantation - a randomized, double-blind trial. Am. J. Transplant, 5: 125-30.

Reverdin, S.G., Bezault, N., Sauvant, D., Bertin, G. 1996. Effects of a probiotic yeast in lactating ruminants: Interaction with dietary nitrogen level, Anim. Feed Sci. Technol., 63, 149-162.

Santini, C., Baffoni, L., Gaggia, F., Granata, M., Gasbarri, R., Gioia, D.D., Biavati, B. 2010. Characterization of probiotic strains: An application as feed additives in poultry against Campylobacter jejuni, Int. J. Food Microbiol. Suppl., (1): 141, 98-108.

Sazawal, S., Hiremath, G., Dhingra, U., Malik, P., Deb, S., Black, R.E. 2006. 
Efficacy of probiotics in prevention of acute diarrhoea: a meta-analysis of masked, randomised, placebo-controlled trials. Lancet Infect. Dis., 6: 374-82.

Schumann, C. 2002. Medical, nutritional and technological properties of lactulose. An update. Eur. J. Nutr., 41(Suppl 1): 1725.

Singh, D., Dimri, A.G., Goyal, P., Chauhan, A., Aggarwal, M.L. and Chacko, K.M. 2012. Microbiological evaluation of street-vended and Restaurant's Food Items. Curr. Res. Biol. Pharmaceutical Sci., 1(1): 25-30.

Soccol1, C.R., Vandenberghe, L.P.S., Spier, M.R., Medeiros, A.B.P., Yamaguishi, C.T., Lindner, J.D., Pandey, A., Soccol, V.T. 2010. The Potential of Probiotics: A Review, Food Technol. Biotechnol., 484: 413-434.

Strompfová, V., Lauková, A., Ouwehand, A.C. 2004. Selection of enterococci for potential canine probiotic additives, Vet. Microbiol., 100, 107-114.

Sýkora, J., Valecková, K., Amlerová, J., et al. 2005. Effects of a specially designed fermented milk product containing probiotic Lactobacillus casei $\mathrm{DN}-114$ 001 and the eradication of $\mathrm{H}$. pylori in children: a prospective randomized double-blind study. J. Clin. Gastroenterol., 39: 692-8.

Tong, J.L., Ran, Z.H., Shen, J., Zhang, C.X., Xiao, S.D. 2007. Meta-analysis: the effect of supplementation with probiotics on eradication rates and adverse events during Helicobacter pylori eradication therapy. Aliment Pharmacol. Ther., 25:155-68.

Uccello, M., Malaguarnera, G., Basile, F., D'agata, V., Malaguarnera, M., Bertino, A., Vacante, M., Drago, F., Uccello, A.B. 2012. Potential role of probiotics on colorectal cancer prevention $B M C$ Surgery, 12(Suppl 1):S35.

Verschuere, L., Rombaut, G., Sorgeloos, P., Verstraete, W. 2000. Probiotic bacteria as biological control agents in aquaculture, Microb. Mol. Biol. Rev., 64 655-671.

Watson, A.K., Kaspar, H., Lategan, M.J., Gibson, L. 2008. Probiotics in aquaculture: The need, principles and mechanisms of action and screening processes, Aquaculture, 274, 1-14.

Yörük, M.A., Gül, M., Hayirli, A., Macit, M. 2004. The effects of supplementation of humate and probiotic on egg production and quality parameters during the late laying period in hens, Poultry Sci., 83: 84-88.

\section{How to cite this article:}

Abhishek Chauhan, Anuj Ranjan, Rupesh Kumar Basniwal and Tanu Jindal. 2016. Probiotic, Prebiotic and Synbiotics in the Prevention of Lifestyle Disorders. Int.J.Curr.Microbiol.App.Sci. 5(12): 933-947. doi: http://dx.doi.org/10.20546/ijcmas.2016.512.102 